\title{
Estimating Chlorophyll Concentration Index in Sugar Beet Leaves Using an Artificial Neural Network
}

\author{
Omer Caliskan ${ }^{1}$, Dursun Kurt ${ }^{1 *}$, Necdet Camas ${ }^{1}$, Mehmet Serhat Odabas ${ }^{2}$ \\ 'Ondokuz Mayis University, Bafra Vocational High School, Department of Crop and Animal Production, \\ Samsun, Turkey \\ ${ }^{2}$ Ondokuz Mayis University, Faculty of Agriculture, Samsun, Turkey
}

Received: 14 May 2018

Accepted: 10 September 2018

\begin{abstract}
The artificial neural network (ANN) method was used in this study for predicting sugar beet (Beta vulgaris L.) leaf chlorophyll concentration from leaves. The experiment was carried out in field conditions in 2015-2016. In this research, symbiotic mychorrhizae as Bio-one (Azotobacter vinelandii and Clostridium pasteurianum) in commercial preparation $(10 \mathrm{~kg} / \mathrm{da})$ and ammonium sulfate $(40 \mathrm{~kg} / \mathrm{da})$ were use used as a fertilizer. In order to measure the leaves' chlorophyll concentration we used a SPAD-502 chlorophyll meter. Artificial neural network, red, green, and blue components of the images were used which was developed to predict chlorophyll concentration. The results showed the ANN model able to estimate sugar beet leaf chlorophyll concentration. The coefficient of determination $\left(\mathrm{R}^{2}\right)$ was found to be 0.98 while mean square error (MSE) was obtained as 0.007 from validation.
\end{abstract}

Keywords: SPAD meter, Beta vulgaris, sugarbeet, artificial neural network, precision agriculture

\section{Introduction}

Diploid and triploid hybrids of sugar beet cultivars can be discerned by leaf morphology [1]. Leaf area and leaf color are determiner in sugar beet for chlorophyll concentration and photosynthesis [2]. The leaves are a very important part of plants concerning some physiological phenomena. In addition, leaves play an important role in some agro-technological practices such as irrigation, fertilization, etc.

*e-mail: dursun.kurt@omu.edu.tr
Traditionally, fertilizers are applied onto the whole field without considering the variation across the land [3]. There are several methods for detecting plant nitrogen content, including plant analysis, leaf chlorophyll measurement, and remote sensing systems. These methods are very time-consuming and expensive approaches and are not suitable for nitrogen applications at different rates. Leaf chlorophyll measurement is a useful tool for predicting crop nitrogen status [4]. Artificial Neural Network (ANN) models are both widely used in many areas for prediction and classification purposes, and it is a computational modeling tool used to solve many complex problems due to its remarkable learning and generalization capabilities [5]. 
Non-destructive methods are found to be more useful when compared with destructive methods [6]. For instance, chlorophyll measurement and remote sensing determine plant nitrogen content, including plant analysis. However, these methods are very time-consuming, costly and unsuitable for nitrogen applications [7], and one of the useful tools for predicting crop nitrogen status is leaf chlorophyll measurement [8]. Using images of the canopy for more confidential information on nitrogen status, a great number of methods have been developed by scientists to estimate the chlorophyll content. To find out the nitrogen levels in plant leaves, image processing has been used [9]. This method used different plants to estimate their yield, yield components and chlorophyll concentration index [10]. To estimate plant nitrogen status by using image processing, digital cameras were used for capturing images. Woebbecke et al. [11] were the first to develop an image processing method to split images into soil and plants. A digital camera system was further developed by Pagola et al. [12] to use plant chlorophyll of winter wheat and barley.

An artificial neural networks model has been widely applied to estimate chlorophyll content of plants. To predict chlorophyll content, artificial neural networks have been applied to non-linear models. Selection of an appropriate technique is very important in obtaining a robust prediction model for leaf chlorophyll content [10]. The results of their study showed the ANN model to be a practical remote sensing system with high accuracy $\left(\mathrm{R}^{2}=0.93\right)$. As for the measurement of chlorophyll content in leaves under field conditions, traditional chlorophyll analysis methods used in a laboratory and some chlorophyll measurements have been developed $[13,14]$. People should be in physical contact with plant leaves in traditional methods, which is both labor- and time-consuming, and this limits the application of these methods in large fields [15]. For a very long period of time, using a camera has been considered to be a practical approach in monitoring leaf chlorophyll content in large fields [16]. The images acquired can give aerial views of the field, such as the chlorophyll content of the crops, and with the help of various computer technologies, they can be identified, categorized, and measured [17]. For instance, a portable color video camera and a personal computer were used by Kawashima and Nakatani [18] for estimating chlorophyll content in leaves. An artificial neural network model was used in the present study to predict of chlorophyll concentration in sugar beet leaves with the help of image processing techniques.

The present research suggested an ANN model to estimate sugarbeet leaf chlorophyll, based on image processing captured with a conventional digital camera.

\section{Material and Methods}

The field research was carried out in the Black Sea Region (N:40.45.026' E:035.41.099'), Turkey in 2011-
2013. Ammonium sulfate (AS) was used as the main fertilizer. Bio-one (Azotobacter vinelandii - ATCC 478 TM and Clostridium pasteurianum - ATCC 6013 TM) was added in ammonium sulfate to use more effectively. Applications were AS + Bio-one and AS- only. A randomized complete block design with 4 replications was used as the experimental design. The air temperature was adjusted to $20^{\circ} \mathrm{C}$ and $23^{\circ} \mathrm{C}$, and the relative humidity was between $40 \%$ and $50 \%$ for day and night, respectively. The leaf chlorophyll concentration index was measured using a chlorophyll meter (Konika Minolta SPAD-502, Soil Plant Analysis Development). A digital camera (DSC-TX7 Sony Corp., Tokyo, Japan) was used to take images from each sampled leaf. The sugar beet leaves were photographed from $40 \mathrm{~cm}$ distance at a lens setting of $9.7 \mathrm{~mm}$ focal length. The resolution of the images was $2048 \times 1536$ pixels and they were recorded in JPEG format. The images contained red, green, and blue components (RGB). Each component had 256 graduations. The image processing was performed by MATLAB software (Matlab R2014a (8.3.0.532) 64-bit). 1200 images were acquired in this experiment.

\section{Image Processing}

Image processing is the application of computer processing techniques on the images made available to it through relevant input mechanisms. Image processing includes acquiring input associated with a digital image, changing the image to suit our needs, acquiring information from the image, which is relevant to us, and producing a desired output. For image processing, the original image of leaves was separated into 3 monochrome images. A sugar beet leaf's reflectance was stronger when compared with its background in images (Fig. 1).
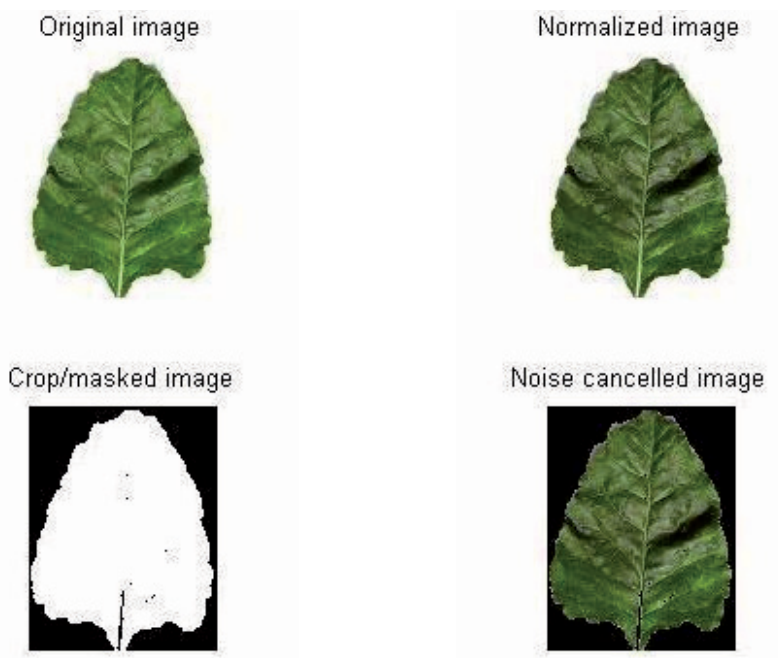

Fig. 1. Step of image processing a) original leaf image, b) normalized image, c) crop/masked image, and d) noise-cancelled image. 
Therefore, the following threshold function was employed to segment the leaf from the background, Eq. 1:

$$
g_{i}(x, y)=\left\{\begin{array}{l}
0 f_{i}(x, y) \leq T_{i} \\
f_{i}(x, y) f_{i}(x, y) \geq T_{i}
\end{array}\right.
$$

...where $g_{i}(x, y)$ is the segmented gray level at pixel $(x, y), \mathrm{f}_{\mathrm{i}}(x, y)$ is the original gray level at pixel $(x, y), \mathrm{T}_{\mathrm{i}}$ is a threshold value and $i$ represent the RGB components. This equation 1 (eq. 1), the pixel values of the leaf are not changed and the pixel values of the background equal zero. In the next step, the sugarbeet leaf reflectance was calculated on the basis of average intensity values of the monochrome images. The average intensity values were calculated as Eq. 2:

$$
A_{i}=\frac{\sum g_{i}(x, y)}{N_{i}}
$$

...where $A_{i}$ is the average intensity value at component $\mathrm{i}, \mathrm{g}_{\mathrm{i}}(\mathrm{x}, \mathrm{y})$ is the intensity value of the pixel representing sugarbeet leaves, $\mathrm{N}_{i}$ is the total number of pixels representing sugarbeet leaves in component $i$, and $i$ represents the RGB components of the image.

In most testing samples, the prediction errors of chl- $b$ were higher than chl- $a$. The reason is that the global threshold values used at the step of the coarse extraction of green pixel are inclined to be blue-green rather than yellow-green. Due to chl- $a$ and chl- $b$ being blue-green and yellow-green, respectively, the prediction performances for chl- $a$ tend to be more accurate than those for chl- $b$ from the plant images, in which the color of the leaves is close to blue-green. In our proposed prediction system, the yellow and the red pigments are considered as noises and removed from the extracted green pixels by image processing.

The prediction errors of chl- $b$ were higher than chl- $a$ in most testing samples since the global threshold values used at the step of the coarse extraction of green pixel are inclined to blue-green rather than yellow-green. The prediction performances for chl- $a$ tend to be more accurate than those for chl- $b$ from the plant images, in which the color of the leaves is close to blue-green, since chl- $a$ and chl- $b$ are blue-green and yellow-green, respectively [19]. The yellow and the red pigments in our prediction system are considered noises and they are removed from the extracted green pixels by image processing.

\section{Artificial Neural Network Model (Multilayer Perceptron Neural Network)}

In order to obtain the model for predicting the chlorophyll content of sugar beet leaves, multilayer perception neural network (MLPNN) was employed. This model was used with a back propagation-learning algorithm and it was developed using the reflectance of sugar beet leaves. RGB components were used as the inputs, and the measured SPAD readings were used as the output. In this research, the MLPNN model consisted of the input layer ( $R, G$, and $B)$ and the output layer (SPAD readings) to match the training data set.

In this study, $70 \%$ of the data set (1200 images) was randomly selected as training data. $15 \%$ of the remaining data set was used for testing, while 15\% was used for validation. Mean square error (MSE) and maximum iteration number were set to 0.001 and 1000 epoch, respectively. Until a satisfactory level of data distribution was reached, the randomization process was repeated because the accuracy of estimation is highly dependent on the dataset. The error of back propagation (BP) training algorithm compares the estimated SPAD values obtained from the model with the corresponding measured SPAD values.

When all weighing indices are fixed and the ANN can accurately estimate the output data [17]. The training error method was used to determine the number of hidden layers and the number of neurons in the hidden layer. In order to evaluate the performance of the ANN model constructed here, mean square error of prediction (MSE) and coefficient of determination $\left(\mathrm{R}^{2}\right)$ were used. Equations 3 and 4 below were used to calculate these criteria:

$$
\begin{gathered}
M S E=\frac{\sum_{i=1}^{N}\left(y_{a i}-y_{p i}\right)^{2}}{N-1} \\
R^{2}=\frac{\left(\sum_{i=1}^{N}\left(y_{a i}-\bar{y}_{a}\right)\left(y_{p i}-\bar{y}_{p}\right)\right)}{\sum_{i=1}^{N}\left(y_{a i}-\bar{y}_{a}\right)^{2}\left(y_{p i}-\bar{y}_{p}\right)^{2}}
\end{gathered}
$$

...where $\mathrm{y}_{\mathrm{i}}$ and $\mathrm{y}_{\mathrm{pi}}$ are actual and predicted leaf SPAD values, respectively, in the equations above. The points taken into consideration there were different

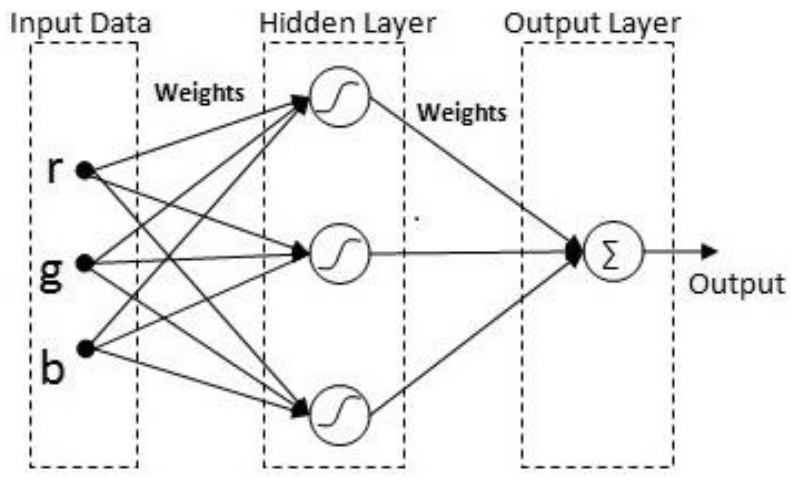

Fig. 2. ANN Model: inputs are red, green, and blue components (RGB); output is SPAD readings. 
transfer functions between layers. In this model, there was 1 hidden layer with 10 neurons. A sigmoid function in the hidden layer and a linear function in the output layer were used in the optimum model obtained from this examination. In order to validate the ANN, $30 \%$ of the data set was used as the input (Fig. 2). The function of the hidden layer was to provide the network's ability to generalize. In building an ANN model, selecting the appropriate number of hidden nodes was very important. Two critical parameters of the network were considering the accuracy and speed of convergence.

\section{Results and Discussion}

The linear relationships between SPAD readings and RGB values across all sampling were found in this study. The coefficient of determination $\left(\mathrm{R}^{2}\right)$ represents the dispersion of the points from the bestfit regression line. Thus, $\mathrm{R}^{2}$ was used to define the best-fit relationship between RGB and SPAD readings estimation (Fig. 3).

The equations of AS + Bio-one ( $\mathrm{y}=0.6617 \mathrm{x}$ $+18.693)$ and $\mathrm{AS}(\mathrm{y}=0.6366 \mathrm{x}+16.37)$ explained $97 \%$ for chl- $a$, and for chl- $b$ of variation in the chlorophyll content of SPAD readings (Fig. 3). The high $\mathrm{R}^{2}$ values showed that chlorophyll meters are an effective tool for estimating chlorophyll concentration in plants. For this reason, SPAD readings data were used in the ANN model.
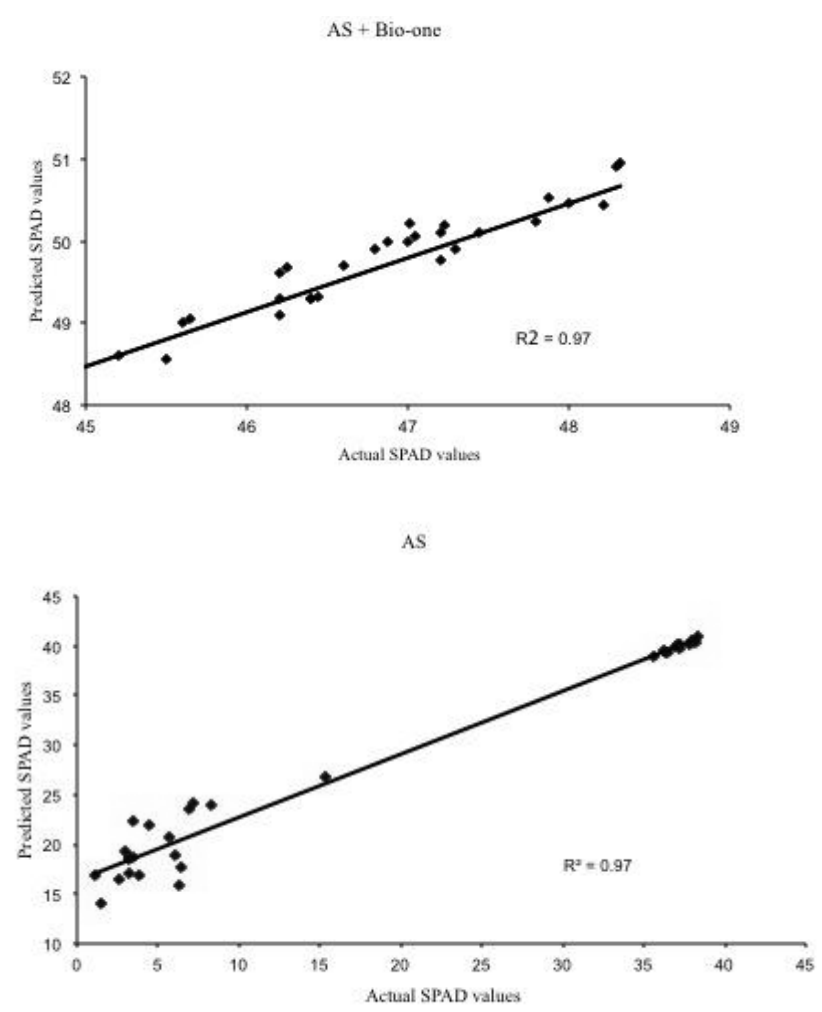

Fig. 3. Relationships between RGB values and SPAD readings.
Our results showed the ANN model trained with the color component (red, green, and blue) to be capable of predicting leaf chlorophyll level $\left(\mathrm{R}^{2}=0.97\right)$. In addition, image processing was shown to be a suitable approach for searching plant chlorophyll level. Leaf chlorophyll contents are proven to be in high correlation with RGB components in plant images by the inputs $(\mathrm{R}, \mathrm{G}$, and $\mathrm{B}$ components) of the ANN model (Fig. 4).

The learning process, which tried to find a balance between RMSE and training time, showed that the number of neurons in this system was determined to be 10. When the results of the ANN model are analyzed, remarkably higher accuracy can be seen when comparing the predicted and actual chlorophyll levels. The mean square error was 0.007 and $\mathrm{R}^{2}$ was 0.97 . The overall average relative error of SPAD readings obtained from the proposed predictor when compared with the experimental measurement was $0.91 \%$ for SPAD readings. The accuracies of prediction in chlorophyll levels and SPAD readings by the ANN-based prediction model are acceptable (Table 1). The linear relationship between the leaf chlorophyll concentrations and color components (RGB) can also be attributed to the artificial neural network model. The results of our study confirmed that the MLPNN model was able to predict sugar beet leaf chlorophyll accurately.

For instance, evaluation of the computer imaging technique for predicting the SPAD readings in potato leaves was investigated by Bohran et al. [20]. In this research facilitating non-contact measurement, a computer-imaging system was devised and evaluated to predict the chlorophyll content in potato leaves. Potato leaves from 15 plants differing in coloration and age were selected for this study. The fitted least square models based on the mean gray scale levels were inversely related to the chlorophyll content of the potato leaf with a $\mathrm{R}^{2}$ of 0.87 using a green band image and with an $\mathrm{R}^{2}$ of 0.79 using a red band image. The prediction model using only mean gray value of the red band showed an average accuracy of $81.6 \%$, with a maximum accuracy of $99.14 \%$.

Analysis of the chlorophyll content of commercial
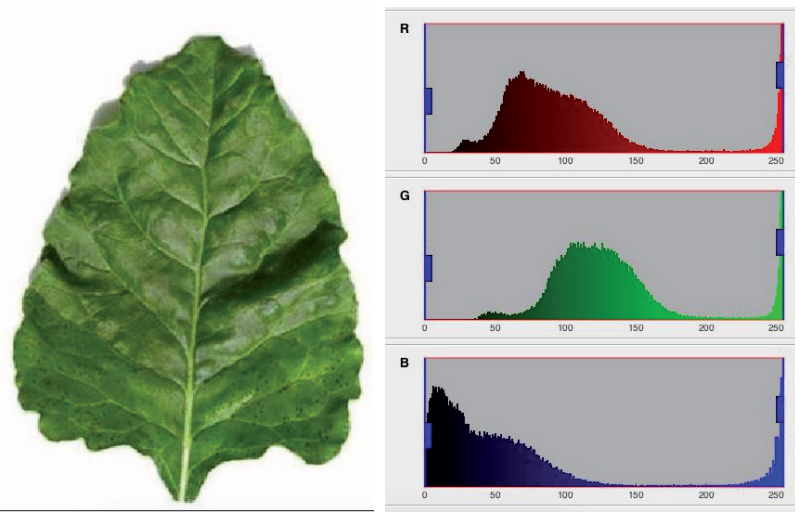

Fig. 4. High correlation with RGB components in sugar beet leaf images. 
Table 1. Comparison of sugar beet leaf chlorophyll contents by the ANN-based predictor and the experimental measurement using a SPAD meter.

\begin{tabular}{|c|c|c|}
\hline Rates of Bio-one & SPAD & $\begin{array}{c}\text { SPAD Relative error } \\
(\%)\end{array}$ \\
\hline AS + Bio-one & $53.12(50.34)$ & 0.97 \\
\hline AS & $37.18(34.35)$ & 0.97 \\
\hline
\end{tabular}

AS, ammonium sulfate; SPAD, soil plant analysis development; the up values are from the ANN-based predictor and the down values in the parentheses are from the experimental measurement

green leafy vegetables was investigated by Limantara et al. [21]. The objective of this study was to evaluate the chlorophyll content of green leafy vegetables found commercially and carry out a comparative investigation between in vivo and in vitro data. In this research, the existence of chlorophylls in 10 green leafy vegetables were reported as SPAD values and percentage $\mathrm{N}$ of the nitrogen meter (in vivo data), as well as total peak area data of HPLC measurement for chlorophyll $a$ and $b$. The results showed that SPAD values and percentage N presented strong linear relationship $\left(R^{2}>0.9\right)$, in which the SPAD-meter performed better detection at very low values.

Vesali et al. [22] developed an android app to estimate chlorophyll content of corn leaves based on contact imaging, which was used for image acquisition from the corn leaves, which captures the light passing through the leaf directly by a smartphone's camera. To estimate SPAD values, various features were extracted from each image. Then superior features were extracted by stepwise regression and sensitivity analysis. The selected features were finally used as inputs to the linear regression and neural network models. The $\mathrm{R}^{2}$ and RMSE values for the linear model were 0.74 and 6.2 , respectively. The corresponding values for the neural network model were 0.82 and 5.10, respectively. Finally, these models were successfully implemented on the SmartSPAD smartphone app. The developed app can be considered a low-cost alternative for estimating chlorophyll content - especially when there is a demand for high availability.

Gholizadeh et al. [23] researched leaf chlorophyll and nitrogen dynamics and their relationship to lowland rice yield for site-specific paddy management. This study proved that the application of a SPAD chlorophyll meter paves the way for real-time paddy $\mathrm{N}$ management and grain yield estimation. Use of a digital camera as an alternative method for non-destructive detection of the leaf chlorophyll content and the nitrogen nutrition status in wheat was investigated by Baresel et al. [24].

Suitable SPAD indices for estimating rice nitrogen status were developed on the basis of the relationships between different SPAD indices calculated from the SPAD readings of various leaf positions and leaf nitrogen and chlorophyll concentrations [25, 26]. Multiple linear regressions' general purpose is to find out more about the relationship between several independent or predictor variables and a dependent or criterion variable. The association between the dependent variable and independent variable(s) is assumed to be linear by a linear regression model. ANN is a non-linear data-driven self-adaptive system; even when the underlying data relationship is not known, it can learn and identify correlated patterns between input data sets and corresponding target values. In the modeling of complex and nonlinear processes and systems, artificial neural networks have begun to be used widely.

\section{Conclusions}

The prediction of chlorophyll concentration index is one of the most important physiological features in agricultural research. In this study, ANN was shown to predict the chlorophyll concentration index of sugar beet leaves by computing the leaf images using image processing, and sugar beet leaves were quantified by SPAD-502 plus chlorophyll meter, which estimates the chlorophyll content of the leaf tissue.

This model was found to be cost-effective and easy to perform when compared with other remote sensing approaches. In addition, an ANN model trained with RGB components was developed in the study, which estimated SPAD readings and the chlorophyll level of the sugar beet leaves with higher accuracy. SPAD measures correlate with leaf photosynthetic efficiency and therefore the health of the plant. This prediction system provides an effective and automatic measurement of chlorophyll to monitor the plant growth status in real time when compared with traditional chlorophyll measurement.

On the other hand, using this ANN model, sugar beet plants can be monitored over time in physiological and quantitative studies. This model is a cost-effective alternative to expensive measurements of leaves by leaf chlorophyll-meters. Therefore, it is concluded that these models may be useful for estimating the chlorophyll concentration index with a high degree of accuracy, and have the potential to be used for structural-functional models of sugar beet.

\section{Acknowledgements}

The authors are grateful to Aydan Ermis and Tuba Odabas (Ondokuz Mayis University, School of Foreign Languages) for editing our English.

\section{Conflict of Interest}

The authors declare no conflict of interest. 


\section{References}

1. TSIALTAS J.M., MASLARIS N. Leaf shape and its relationship with leaf area index in a sugar beet (Beta vulgaris L.) cultivar. Photosynthetica, 45 (4), 527, 2007. http://dx.doi.org/10.1007/s11099-008-0009-9

2. KESSLER S., SINHA N. Shaping up: the genetic control of leaf shape. Current Opinion in Plant Biology, 7, 65, 2005. http://dx.doi.org/10.1016/j.pbi.2003.11.002

3. TEMIZEL K.E., ODABAS M.S., SENYER N., KAYHAN G., BAJWA S., CALISKAN O., ERGUN E. Comparision of some models for estimation of reflectance of hypericum leaves under stress conditions. Central European Journal of Biology, 9 (12), 1226, 2014. https://doi.org/10.2478/ s11535-014-0356-4.

4. ODABAS M.S., TEMIZEL K.E., CALISKAN O., SENYER N., KAYHAN G., ERGUN E. Determination of reflectance values of hypericum's leaves under stress conditions using adaptive network based fuzzy inference system. Neural Network World, 24 (1), 79, 2014. http:// dx.doi.org/10.14311/nnw.2014.24.004

5. MARKWELL J., OSTERMAN J.C., MITCHELL J.L. Calibration of the minolta SPAD-502 leaf chlorophyll meter. Photosynthesis Research, 16, 467-472, 2005. https:// dx.doi.org/10.1007/BF00032301

6. GAUTAM R.K., PANIGRAHI S. Leaf nitrogen determination of corn plant using aerial images and artificial neural networks. Canadian Biosystems Engineering, 49, 71, 2007.

7. NOH H., ZHANG Q., SHIN B., HAN S., FENG L. A neural network model of maize crop nitrogen stress assessment for a multi- spectral imaging sensor. Biosystems Engineering, 94, 477, 2006. http://dx.doi. org/10.1016/j.biosystemseng.2006.04.009

8. ODABAS M.S., LEELARUBAN N., SIMSEK H., PADMANABHAN G. Quantifying impact of droughts on barley yield in North Dakota, USA using multiple linear regression and artificial neural network. Neural Network World, 24 (4), 343, 2014. http://dx.doi.org/10.14311/ NNW.2014.24.020

9. ODABAS M.S., KAYHAN G., ERGUN E., SENYER N. Using artificial neural network and multiple linear regression for predicting the chlorophyll concentration index of Saint John's wort leaves. Communications in Soil Science and Plant Analysis, 47 (2), 237, 2016. http://dx.doi. org/10.1080/00103624.2015.1104342.

10. SIMSEK H., CEMEK B., ODABAS M.S., RAHMAN $S$. Estimation of nutrient concentrations in runoff from beef cattle feedlot using adaptive neuro-fuzzy inference systems. Neural Network World, 5 (15), 501, 2015. http:// dx.doi.org/10.14311/nnw.2015.25.025.

11. WOEBBECKE D.M., MEYER G.E., BARGEN K.V., MORTENSEN D.A. Color indices for weed identification under various soil, residue and lighting conditions. Transactions of the ASAE, 38, 259, 1995. http://dx.doi. org/10.13031/2013.27838.

12. PAGOLA M., RUBEN O., IGNACIO I., HUMBERTO B., EDURNE B., PEDRO A.T., CARMEN L., BERTA L. New method to assess barley nitrogen nutrition status based on image color analysis comparison with SPAD-502. Computers and Electronics in Agriculture, 65, 213, 2009. http://dx.doi.org/10.1016/j.compag. 2008.10.003.

13. AZADEH A., SABERI M., ASADZADEH S.M. An adaptive network based fuzzy inference system-auto regression-analysis of variance algorithm for improvement of oil consumption estimation and policy making: The cases of Canada, United Kingdom, and South Korea. Applied Mathematical Modelling, 35 (2), 581, 2011. https:// doi.org/10.1016/j.apm.2010.06.001

14. COSTE S., BARALOTO C., LEROY C., MARCON E., RENAUD A., RICHARDSON A.D., ROGGY J.C., SCHIMANN H. UDDLING J. HERAULT B. Assessing foliar chlorophyll contents with the SPAD-502 chlorophyll meter: a calibration test with thirteen tree species of tropical rainforest in French Guiana. Annals of Forest. Science. 67, 607, 2010. doi: 10.1051/forest $/ 2010020$

15. ODABAS M.S., SIMSEK H., LEE C.W., ISERI I. Multilayer Perceptron Neural Network Approach to Estimate Chlorophyll Concentration Index of Lettuce (Lactuca sativa L.). Communications in Soil Science and Plant Analysis, 48 (2), 162, 2017. https://doi.org/10.1080/00 103624.2016.1253726

16. HABOUNDANE D.D., TREMBLAY N., MILLER J.R. VIGNEAULT P. Remote Estimation of Crop Chlorophyll Content Using Spectral Indices Derived From Hyperspectral Data. IEEE Transactions On Geoscience And Remote Sensing, 46 (2), 423, 2008. Doi: 10.1109/ TGRS.2007.904836

17. POMPELLI M.F., FRANCA S.C., TIGRE R.C., DE OLIVEIRA M.T. SACILOT M., PEREIRA E.C. Spectrophotometric determinations of chloroplastidic pigments in acetone, ethanol and dimethylsulphoxide. Brazilian Journal of Biosciences, 11 (1), 52, 2013. http://www.ufrgs.br/seerbio/ojs/index.php/rbb/article/ view/2281

18. KAWASHIMA S., NAKATANI M. An algorithm for estimating chlorophyll content in leaves using a video camera. Annals of Botany, 81, 49, 1998. https://doi. org/10.1006/anbo.1997.0544

19. PENG Y., ROBERTSON A.N., ARKEBAUER T., GITELSON A.A. Assessment of Canopy Chlorophyll Content Retrieval in Maize and Soybean: Implications of Hysteresis on the Development of Generic Algorithms. Remote Sensing, 9 (3), 226, 2017. doi:10.3390/ rs9030226

20. BOHRAN M.S., PANIGRAHI S., SATTER M.A., GU H. Evaluation of computer imaging technique for predicting the SPAD readings in potato leaves. Information Processing in Agriculture, 4 (4), 275, 2017

21. LIMANTARA L., DETTLING M., INDRAWATI R., BROTOSUDARMO T.H.P. Analysis on the Chlorophyll Content of Commercial Green Leafy Vegetables. Procedia Chemistry, 14, 225, 2015.

22. VESALI F., OMID M., KALEITA A., MOBLI H. Development of an android app to estimate chlorophyll content of corn leaves based on contact imaging. Computers and Electronics in Agriculture, 116, 211, 2015

23. GHOLIZADEH A., SABERIOON M., BORUVKA L., WAYAYOK A., SOOM M.A.M. Leaf chlorophyll and nitrogen dynamics and their relationship to lowland rice yield for site-specific paddy management. Information Processing in Agriculture. 4 (4), 259, 2017.

24. BARESEL J.P., RISCHBECK P., HU Y., KIPP S., SCHMIDHALTER U. Use of a digital camera as alternative method for non-destructive detection of the leaf chlorophyll content and the nitrogen nutrition status in wheat. Computers and Electronics in Agriculture, 140, 25, 2017. 
25. LIN F.F., QIU L.F., DENG J.S., SHI Y.Y., WANG K. Investigation of SPAD meter-based indices for estimating rice nitrogen status.Computers and Electronics in Agriculture, 71 (1), 60, 2010.
26. DEY F.F.F., A.K., SHARMA M., MESHRAM M.R. An Analysis of Leaf Chlorophyll Measurement Method Using Chlorophyll Meter and Image Processing Technique. Procedia Computer Science. 85, 286, 2016. 
